\title{
Clinical follow-up study of 166 cases of children with hypertension
}

\author{
Zhenquan Wang, Yiping Shao, Jiahui Jin, Xing Rong, Huixian Qiu, Rongzhou Wu, Maoping Chu \\ Children's Heart Center, The Second Affiliated Hospital and Yuying Children's Hospital, Wenzhou Medical University, Wenzhou, Zhejiang, China \\ Contributions: (I) Conception and design: Z Wang, Y Shao; (II) Administrative support: R Wu, M Chu; (III) Provision of study materials or patients: \\ X Rong, H Qiu, M Chu; (IV) Collection and assembly of data: Y Shao, Z Wang; (V) Data analysis and interpretation: Z Wang, Y Shao, J Jin; (VI) \\ Manuscript writing: All authors; (VII) Final approval of manuscript: All authors. \\ Correspondence to: Maoping Chu. Children's Heart Center, The Second Affiliated Hospital and Yuying Children's Hospital, Wenzhou Medical \\ University, No. 109 Xueyuan Xi Road, Wenzhou, Zhejiang 325027, China. Email: chmping@hotmail.com.
}

\begin{abstract}
Background: Childhood hypertension is a challenge for pediatricians to discover and diagnose. We sought to analyze its clinical characteristics and related risk factors in patients at a single center.

Methods: From 2009 to 2019, 166 children with hypertension were retrospectively analyzed, and their clinical manifestations and relevant laboratory data were collected for statistical analysis.

Results: A total of 120 males and 46 females were included in this study. Males were more common than females $(\mathrm{P}=0.012)$, and $86.7 \%$ were from rural areas. Hypertension appeared in all age groups, but most of them were puberty (52.4\%). Most primary hypertension cases (57/91) had no obvious clinical symptoms, and BMI (OR 1.085, 95\% CI: 1.004-1.173, P=0.038) and a family history of hypertension (OR 5.605, 95\% CI: 2.229-14.092, $\mathrm{P}<0.001)$ were the risk factors. In the 75 secondary hypertension cases, renal hypertension $(62.7 \%)$ was the main cause and headache and dizziness were the most common symptoms, and the serum urea is a risk factor (OR 1.524, 95\% CI: 1.037-2.239, $\mathrm{P}=0.032$ ).

Conclusions: BMI and a family history of hypertension were associated with primary hypertension. The serum urea was related to secondary hypertension. Emphasis on family history, strengthening family health management and education and publicity of hypertension, were important for diagnosis and detection of children with hypertension.
\end{abstract}

Keywords: Childhood hypertension; regional distribution; sex; risk factors

Submitted Dec 14, 2020. Accepted for publication May 24, 2021.

doi: $10.21037 /$ tp-20-446

View this article at: http://dx.doi.org/10.21037/tp-20-446

\section{Introduction}

Hypertension has long been perceived as a disease of adults, rarely seen in children and adolescents, though recent reports reveal it has become a major public health problem in children worldwide (1). The prevalence of hypertension in children and adolescents reaches $3 \%$ to $6 \%$, while the total prevalence of obese adolescents has increased nearly five times, reaching about $30 \%$ (2). Equally concerning, hypertension is a lifelong disease; childhood hypertension is one of the most important risk factors for cardiovascular disease in adulthood, causing damage to target organs and worsening with age (3). Although the prevalence of hypertension in children is increasing, many children with hypertension are misdiagnosed or not diagnosed.

Numerous studies have confirmed that elevated blood pressure in childhood is associated with increased risk of hypertension and metabolic diseases in adults (4). As the time frame of hypertension diagnosis could influence outcome of children in terms of growth development and prognosis, it is critical to make an early diagnosis and draw up treatment and management measures as soon as possible. This study sought to analyze the epidemiological distribution of hypertension in children in Wenzhou, China, determine its clinical characteristics, evaluate the possible risk factors related to its incidence, and evaluate whether these characteristics can provide clinical guidance. We present the following article in accordance with the 
STROBE reporting checklist (available at https://dx.doi. org/10.21037/tp-20-446).

\section{Methods}

\section{Participants}

From 2009 to 2019,166 children $(0<$ age $<18)$ were diagnosed with hypertension in Yuying Children's Hospital, the Second Affiliated Hospital of Wenzhou Medical University. These patients were divided into two groups: primary hypertension group (91 cases) and secondary hypertension group (75 cases).

\section{Methods}

This is a retrospective study. The following data were collected for each patient: (I) epidemiological and clinical characteristics including age, gender, regional distribution, height, weight, body mass index (BMI); (II) related laboratory indicators: urinary protein, triglycerides, total cholesterol, high-density lipoprotein cholesterol, lowdensity lipoprotein cholesterol; and (III) data from special examinations such as echocardiography, electrocardiogram, or abdominal echocardiography.

All procedures performed in this study involving human participants were in accordance with the Declaration of Helsinki (as revised in 2013). The study was approved by the ethics committee of The Second Affiliated Hospital and Yuying Children's Hospital (LCKY2018-79). Individual consent for this retrospective analysis was waived.

\section{Inclusion criteria}

On the basis of the clinical practice guidelines for children with hypertension developed by the American Academy of Pediatrics in 2017 (1) and the updated Chinese hypertension diagnosis guidelines (5), patients were included if they had hypertension, defined in children aged $1-13$ years as average clinic measured SBP and/or DBP $\geq 95$ th percentile (on the basis of age, sex and height percentile); and in children over 13 years old as $\geq 130 / 80 \mathrm{mmHg}$. Patients with white coat hypertension (WCH, it refers to the scenario in which BPs are elevated in the office but normal outside the office) (6), transient hypertension, or postoperative hypertension were excluded from the analysis.

\section{Follow-up process}

Long-term outpatient follow-up after discharge is recommended for all children. For various reasons, children who are unable to follow-up with our clinic are advised to visit local community hospitals or health clinics regularly. At the same time, we conducted an online survey for all children on the status and results of follow-up visits. If the condition changes, it is recommended to go to our hospital for further treatment.

\section{Statistical analysis}

IBM SPSS Statistics ${ }^{\circledR}$ version 22.0 software (Armonk, NY, USA) was used for these analyses. In the data, continuous variables were described as mean \pm standard deviation or median; categorical variables were percentages of the number of cases. In the univariate analysis, the chi-square test and Mann-Whitney $\mathrm{U}$ test were used to screen for related factors of the two groups, and logistic regression analysis was used to determine risk factors for primary and secondary hypertension. Differences with $\mathrm{P}<0.05$ were considered statistically significant.

\section{Results}

Age and gender distribution characteristics of children with bypertension

As Figure 1 depicts, from 2009 to 2019, our centers diagnosed hypertension in 166 children, including 120 males and 46 females. There were more males than females in both primary hypertension $(\mathrm{M}: \mathrm{F}$ ratio $=4.06: 1)$ and secondary hypertension $(\mathrm{M}: \mathrm{F}=1.68: 1)(\mathrm{P}=0.012)$. As shown in Table 1 and Figure 2, the age of the children ranged from 3 months to 17 years, with a median age of 13 years. The distribution of primary hypertension or secondary hypertension is mainly puberty $(59.3 \%$ and $44.0 \%$, respectively). And $39.2 \%$ (65/166) of the total population were pubertal males. Most of the children with primary hypertension had a family history of hypertension $(53.8 \%$ vs. $16.0 \%, \mathrm{P}<0.001)$. And most primary hypertension was found after a health examination $(53.8 \%$ vs. $10.7 \%$, $\mathrm{P}<0.001)$.

\section{Regional distribution characteristics of children with bypertension}

The Second Affiliated Hospital of Wenzhou Medical University, serving the central city and beyond. Only $13.3 \%$ of the children came from Wenzhou central city, with the 


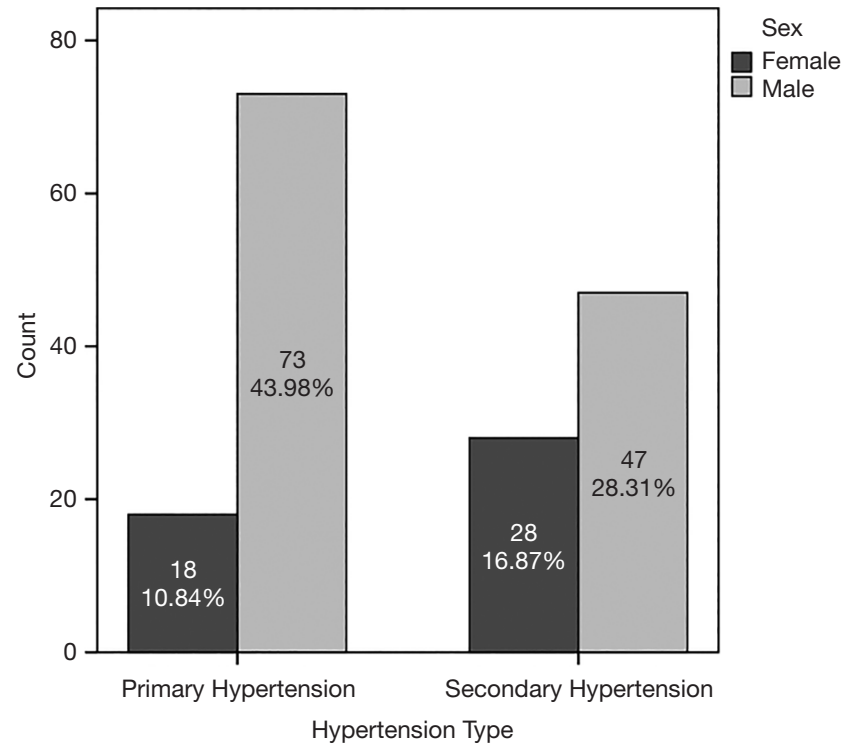

Figure 1 Comparison of sex between primary hypertension and secondary hypertension.

majority $(86.7 \%)$ coming from rural areas in Wenzhou $(71.7 \%)$ and outside Wenzhou (15.0\%). This distribution is shown in Figure 3.

\section{Clinical characteristics and special examination of children with hypertension}

\section{Clinical manifestations of children with primary} hypertension

At the routine physical examination, 50/91 (54.9\%) children with primary hypertension were asymptomatic. Among the symptomatic children, the most common clinical symptoms were dizziness or headache $(36 / 40)$, which was generally most noticeable after getting up in the morning. There were 11 cases with chest tightness (2 cases) and palpitation (10 cases). And other symptoms are less common, such as blurred vision ( 2 cases) and syncope (1 case).

\section{Clinical manifestation in children with secondary hypertension}

Only 15/75 (20.0\%) children with secondary hypertension were asymptomatic. Headaches and/or dizziness (28 cases) were the most common symptoms in 61 of children with secondary hypertension. Digestive system symptoms (24 cases) include abdominal pain and vomiting; secondly, 15 cases of convulsion and 5 cases of edema; 1 patient had cardiovascular system involvement including increased heart rate, chest tightness.

\section{Etiology of secondary hypertension in children}

As shown in Figure 4, in 75 cases of secondary hypertension, renal hypertension was the primary cause, mainly related to kidney diseases such as glomerulonephritis and chronic renal failure, accounted for $62.7 \%$. Hypertension induced by connective tissue disease was the secondary cause (12.0\%). Secondly, endocrine hypertension, accounted for $10.7 \%$, cardiovascular hypertension about $9.3 \%$, and the remainder included neurosystemic hypertension $(2.70 \%)$ and drug-induced hypertension (2.70\%).

\section{Follow up of children with primary and secondary bypertension}

Subsequently, all 166 cases of children diagnosed with hypertension were followed up, as shown in Table 2. In primary hypertension, 29 children kept their blood pressure within the normal range after discharge from hospital, 25 of whom were not taking antihypertensive drugs, and in 4 who were. However, there were also 28 children with poorly controlled blood pressure, 12 of whom were taking antihypertensive drugs, and 16 of them did not take medication. Among those with secondary hypertension, 25 children had controlled blood pressure, of whom 15 were not taking antihypertensive drugs, and 10 took antihypertensive drugs to lower their blood pressure. However, 10 children failed to control their blood pressure even after taking antihypertensive drugs. Finally, 63 cases did not receive regular monitoring and follow-up (33 cases of primary hypertension and 30 cases of secondary hypertension). Ten children were lost to contact.

\section{Comparison of different indices of childhood hypertension}

As shown in Table 3, the BMI of children with primary hypertension was significantly higher than in those with secondary hypertension $(23.2 \pm 5.52$ vs. $20.5 \pm 6.48, \mathrm{P}=0.004)$, while triglycerides $(1.14$ vs. $1.38 \mathrm{mmol} / \mathrm{L}, \mathrm{P}=0.026)$, total cholesterol (4.01 vs. $4.43 \mathrm{mmol} / \mathrm{L}, \mathrm{P}=0.001)$, low-density lipoprotein cholesterol ( 2.15 vs. $2.56 \mathrm{mmol} / \mathrm{L}, \mathrm{P}=0.002)$ and serum urea $(4.06$ vs. $4.79 \mathrm{mmol} / \mathrm{L}, \mathrm{P}<0.001)$ were significantly lower in those with secondary hypertension. Logistic analysis was performed for the correlation between primary hypertension and secondary hypertension (Table 4). The results showed that male (OR 2.416, 95\% CI: $1.204-$ 4.848, $\mathrm{P}=0.013$ ), age (OR 1.093, 95\% CI: 1.006-1.187, 
Table 1 General information of children

\begin{tabular}{lcc}
\hline & Primary hypertension & Secondary hypertension \\
\hline Total count & 91 & 75 \\
Sex & & $47(62.7 \%)$ \\
Male & $73(80.2 \%)$ & $28(37.3 \%)$ \\
Female & $18(19.8 \%)$ & \\
Age distribution & & $1(1.3 \%)$ \\
Infancy $(<1$ y) & 0.012 \\
Early childhood $(1-3$ y) & $3(3.3 \%)$ & $7(9.3 \%)$ \\
Preschool $(4-6$ y) & $4(4.4 \%)$ & $5(6.7 \%)$ \\
School age $(7-12$ y) & $30(33.0 \%)$ & $29(38.7 \%)$ \\
Puberty $(\geq 13$ y) & $54(59.3 \%)$ & $33(44.0 \%)$ \\
Health examination & $49(53.8 \%)$ & $8(10.7 \%)$ \\
Family history of hypertension & $49(53.8 \%)$ & $12(16.0 \%)$ \\
\hline
\end{tabular}

Data are presented as $\mathrm{N}(\%)$.

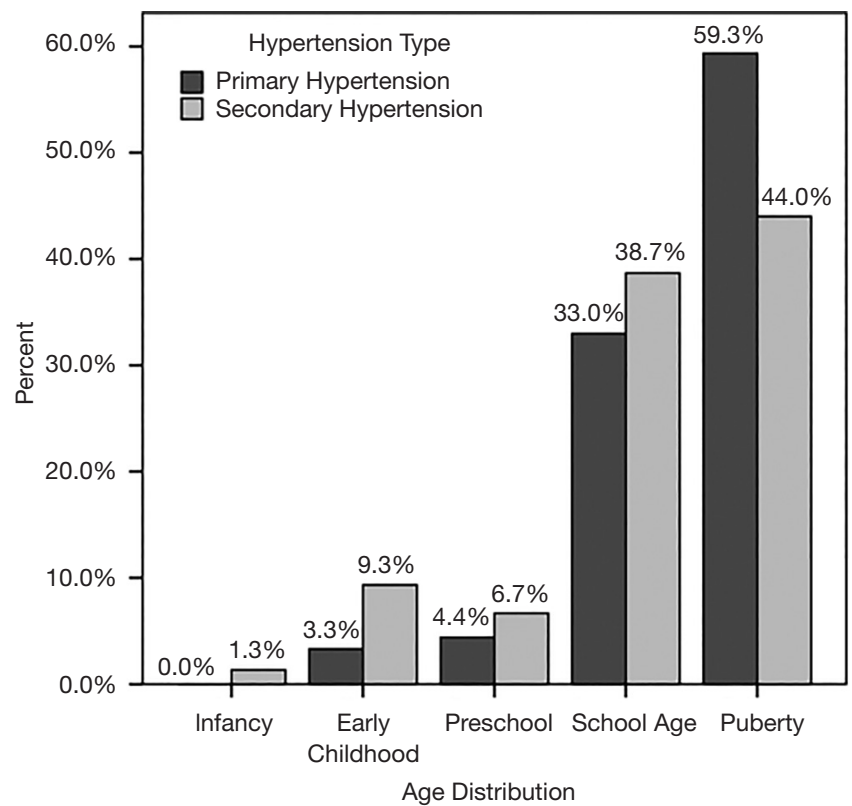

Figure 2 Comparison of age distribution between primary hypertension and secondary hypertension.

$\mathrm{P}=0.035)$, BMI (OR 1.083, 95\% CI: 1.024-1.145, $\mathrm{P}=0.005)$ and a family history of hypertension (OR 6.125, 95\% CI: $2.916-12.867, \mathrm{P}<0.001)$ were risk factors for primary hypertension. When we adjusted for confounding factors, such as age, sex, BMI (OR 1.085, 95\% CI: 1.004-1.173, $\mathrm{P}=0.038$ ) and a family history (OR 5.605, 95\% CI:

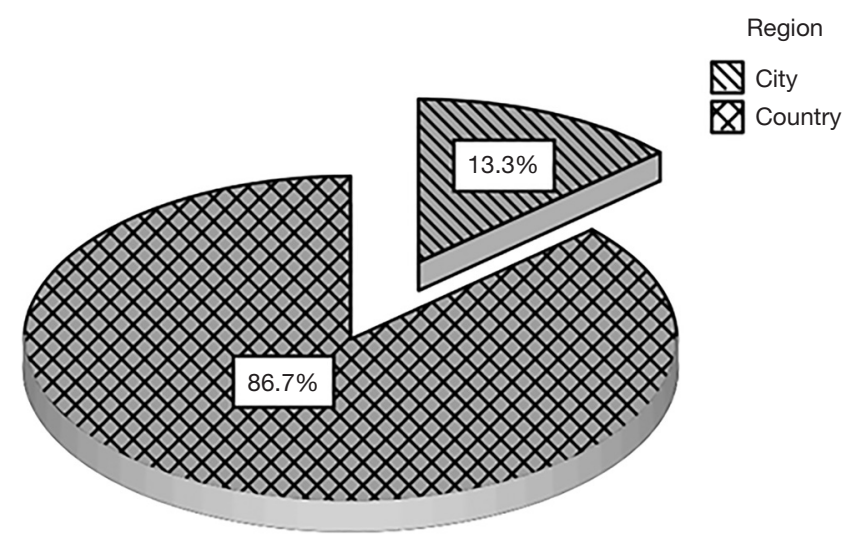

Figure 3 Characteristics of regional distribution.

2.229-14.092, $\mathrm{P}<0.001)$ remained the risk factor for primary hypertension. We also analyzed the risk factors for secondary hypertension, and the results showed that total cholesterol (OR 2.123, 95\% CI: 1.393-3.236, P<0.001), low density lipoprotein cholesterol (OR 2.156, 95\% CI: $1.312-3.544, \mathrm{P}=0.002$ ), serum urea (OR $1.438,95 \% \mathrm{CI}$ : $1.163-1.779, \mathrm{P}=0.001)$ and creatinine (OR 1.008, 95\% CI: $1.001-1.015, \mathrm{P}=0.023)$ were risk factors for secondary hypertension. And after adjusting for confounders such as age and sex, as shown in Table 5, serum urea is still a risk factor for secondary hypertension (OR 1.524, 95\% CI: $1.037-2.239, \mathrm{P}=0.032)$.

\section{Discussion}


In this study, we analyzed the epidemiological distribution of hypertension in children, determined its clinical characteristics, evaluated the possible risk factors related to its incidence. In this study, we found primary hypertension to be more common than secondary hypertension in children, and renal diseases were the most common in secondary hypertension. A family history of hypertension and increased BMI were related to primary hypertension, and elevated LDL-C and serum urea were associated with secondary hypertension. For children with elevated blood pressure or hypertension, the history and family history should be inquired carefully, and blood pressure should be checked regularly.

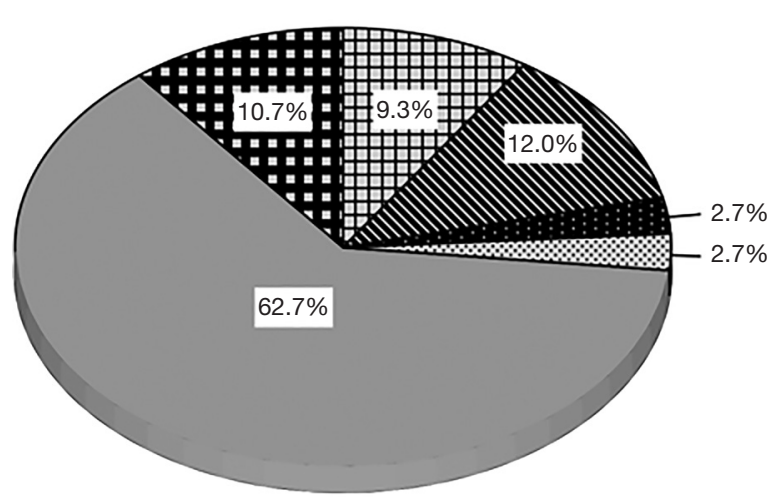

Category

田 Cardiovascular Hypertension

N Hypertension induced by Connective Tissue Disease

:: Drug-induced Hypertension

Nurosystemic Hypertension

$\square$ Renal Hypertension

E. Endocrine Hypertension

Figure 4 Characteristic of etiology of secondary hypertension.
Hypertension is classified into two types: primary and secondary, according to the etiology. Primary hypertension refers to disease with elevated blood pressure for which the cause is unknown; while the cause of secondary hypertension is clear, hypertension is only one of the clinical manifestations of an underlying disease. Previous studies (7) have suggested that secondary hypertension is the main cause of hypertension in children and adolescents. However, in 2017, the American Academy of Pediatrics "Clinical Practice Guidelines For Screening And Management Of Hypertension In Children And Adolescents" found that primary hypertension was becoming more prevalent than secondary hypertension (3). Our 10-year retrospective study found 91 cases of primary hypertension versus 75 cases of secondary hypertension, consistent with the American Academy of Pediatrics findings. With the increasing rate of obesity in children, primary hypertension has become one of the most common diseases in children $(8,9)$.

In our study, hypertension more commonly affected male patients than female patients, regardless of whether it was primary or secondary hypertension, showing significant differences between sex. These differences, consistent with reports in adult patients (10), may be related to differences in living habits, lifestyle, diet, and environmental and other factors between men and women. In addition, as reported in other studies (11), the distribution of hypertension skewed toward adolescents, and the overall trend of the number of patients increased with age (12). The increase in the number of adolescent patients with hypertension is not only related to the period of rapid growth and development, but also to sex hormones, growth hormones and cortisol, making adolescent children more likely than other age groups to present with hypertension $(4,13)$.

Health education is very crucial to discover and follow

Table 2 Follow up of children with primary and secondary hypertension

\begin{tabular}{lccc}
\hline & \multicolumn{2}{c}{ Follow-up } \\
\cline { 2 - 4 } & Primary hypertension (N) & Secondary hypertension (N) & Total count \\
\hline Good blood pressure control, no antihypertensive drugs & 25 & 15 & 10 \\
Good blood pressure control is OK, take antihypertensive \\
drugs
\end{tabular}


Table 3 comparison of different indexes between primary and secondary hypertension

\begin{tabular}{|c|c|c|c|}
\hline & Primary hypertension & Secondary hypertension & $P$ value \\
\hline BMI & $23.2 \pm 5.52$ & $20.5 \pm 6.48$ & 0.004 \\
\hline Triglyceride (mmol/L) & $1.14(0.835-1.73)$ & $1.38(0.97-2.18)$ & 0.026 \\
\hline Total cholesterol (mmol/L) & $4.01(3.53-4.58)$ & $4.43(3.98-5.40)$ & 0.001 \\
\hline Low density lipoprotein cholesterol (mmol/L) & $2.15(1.85-2.64)$ & $2.56(2.15-3.22)$ & 0.002 \\
\hline Serum urea $(\mathrm{mmol} / \mathrm{L})$ & $4.06(3.49-4.61)$ & $4.79(3.52-8.18)$ & $<0.001$ \\
\hline Creatinine $(\mu \mathrm{mol} / \mathrm{L})$ & $53.2(42.9-63.7)$ & $55.0(34.3-123)$ & 0.681 \\
\hline Urea/creatinine ratio & $0.08(0.06-0.09)$ & $0.08(0.06-0.12)$ & 0.325 \\
\hline
\end{tabular}

Data are presented as mean \pm SD or median (Q1-Q3). BMI, body mass index.

Table 4 Multiple regression analysis of primary hypertension

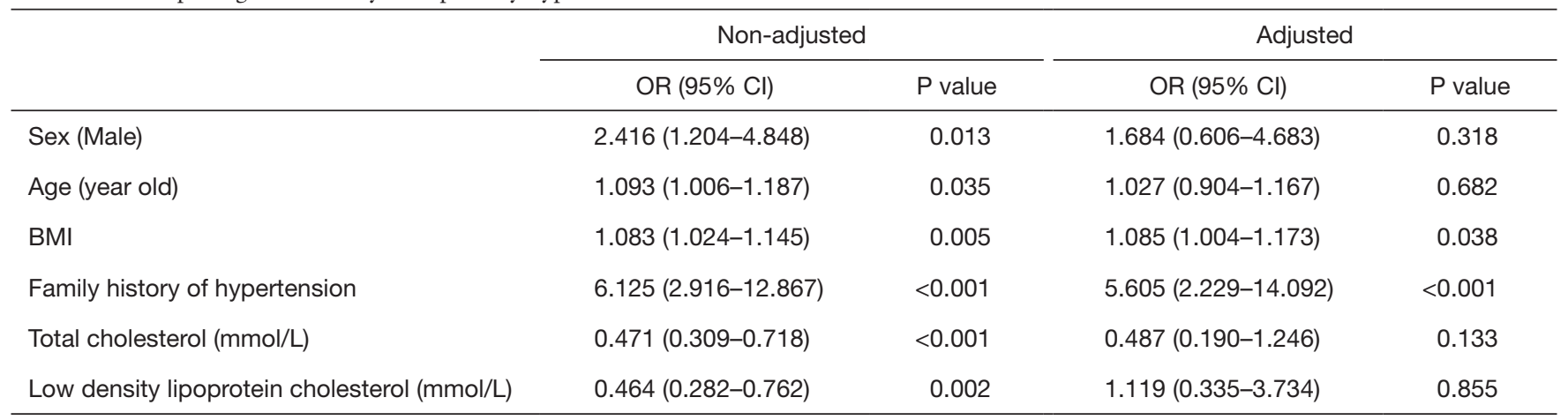

Statistic: odds ratio (95\% confidence interval)/OR (95\% Cl). BMI, body mass index.

Table 5 Multiple regression analysis of secondary hypertension

\begin{tabular}{|c|c|c|c|c|}
\hline & \multicolumn{2}{|c|}{ Non-adjusted } & \multicolumn{2}{|c|}{ Adjusted } \\
\hline Sex (Male) & $0.414(0.206-0.830)$ & 0.013 & $0.539(0.201-1.446)$ & 0.220 \\
\hline Age (year old) & $0.915(0.843-0.994)$ & 0.035 & $0.863(0.742-1.004)$ & 0.056 \\
\hline Total cholesterol (mmol/L) & $2.123(1.393-3.236)$ & $<0.001$ & $1.228(0.458-3.295)$ & 0.683 \\
\hline serum urea (mmol/L) & $1.438(1.163-1.779)$ & 0.001 & $1.524(1.037-2.239)$ & 0.032 \\
\hline Creatinine $(\mu \mathrm{mol} / \mathrm{L})$ & $1.008(1.001-1.015)$ & 0.023 & $1.006(0.981-1.031)$ & 0.630 \\
\hline
\end{tabular}

Statistic: odds ratio (95\% confidence interval)/OR (95\% Cl). 
up hypertension. Firstly, in this study, we found that most $(86.7 \%)$ of the children with hypertension came from lessdeveloped rural areas, while only $13.3 \%$ came from central cities. This is consistent with some findings reported in the literature $(14,15)$. The lack of knowledge and education and the significant differences in cultural practices, living habits, living environments, and sources of information between urban and rural areas, these may be the reasons why there are more patients in rural areas than in urban areas (16).

Secondly, most of the children with primary hypertension (50/91) had no obvious symptoms and manifestations. Among these children, most of symptoms are mild or sporadic and can often be overlooked. This is also one of the reasons for the low rates of awareness and treatment of hypertension in children. On the one hand, children with hypertension have no obvious clinical signs in the early stage when it has not yet caused structural changes in the body organs (17). On the other hand, it also shows that society and families pay insufficient attention to the problem of hypertension in children, and lack of knowledge and education about hypertension in children. Thirdly, it is also indirectly supported by the phenomenon that abnormal blood pressure in 50 asymptomatic children was detected through a health examination in a medical institution rather than through home health monitoring. At last, we found that 76/166 patients had poor follow-up compliance after discharge, including casual withdrawal of medication, without monitoring of blood pressure changes, and irregular follow-up. These outcomes may be related to the following factors: asymptomatic hypertension, lack of attention to health knowledge, insufficient health education and publicity of hypertension. The above highlights the importance of health education and publicity. These views are similar to those reported in the literature (18). All of above showed the lack of knowledge, health education and publicity may be the risk factors of hypertension in children. The task of preventing hypertension is still very difficult (18).

From our study, BMI and a family history of hypertension were risk factors of primary hypertension. In parallel with increasing BMI, the prevalence of hypertension has been reported increased among children. Although BMI is the main factor responsible for increasing rate of hypertension among adolescent, genetic, and family history, susceptibility should not be ignored (19). Of course, previous studies also have found that preterm birth, birth by cesarean section, and family history of hypertension are risk factors for primary hypertension in children $(20,21)$. Unfortunately, the birth history of these children has not been investigated in this study. And further investigation could be carried out in the later period. Furthermore, after controlling for confounding factors, we found that serum urea is a risk factors for secondary hypertension in this study. Renal diseases are the most common secondary hypertension in children (22). High blood pressure is an important factors of impaired kidney function and kidney damage. Long-term hypertension affects renal function, which leads to serum urea filtration and reabsorption dysfunction (23). Elevated urea is a danger signal of kidney damage which could cause hypertension.

There are also limitations in this study. First, our study sample size is small, and the time span is long. Although we conducted active follow-up, some cases were nonetheless lost to follow-up, leading to unsatisfactory results of our follow-up, for example, only $54.2 \%$ of the cases entered the follow-up results. In addition, our study was limited to the Wenzhou region and may not be broadly applicable, as we were limited in our ability to deeply investigate the birth history, growth and development history, families' specific economic situation, lifestyle habits, physical exercise or mental health, all of which may affect blood pressure results.

To sum up, it is difficult for pediatricians to diagnose and observe the therapeutic effect of primary hypertension in children. First, the clinical symptom is mild or asymptomatic; in addition, awareness of hypertension is not enough. We believe that all of individuals, families and medical community should pay attention to children's hypertension. Social and medical institutions should actively promote the establishment of children's health management systems and improve family monitoring and follow-up systems.

Most children with hypertension have no obvious symptoms or manifestations, making it difficult to diagnose. However, the increase in incidence rate of hypertension in children shows the importance of enhancing our education and awareness of children's hypertension and strengthening family management, monitoring and health education. Our findings also show the importance of taking steps toward early discovery of primary hypertension and detection of secondary hypertension. BMI and A family history of hypertension were associated with primary hypertension. The serum urea was related to secondary hypertension. Emphasis on family history, strengthening family health management and education and publicity of hypertension, were important for diagnosis and detection of children with hypertension.

\section{Conclusions}


In this study, we found primary hypertension to be more common than secondary hypertension in children, and renal diseases were the most common in secondary hypertension. BMI and a family history of hypertension were related to primary hypertension. And serum urea was a risk factor for secondary hypertension. For children with elevated blood pressure or hypertension, the family history should be carefully inquired, the root cause should be traced, and a careful physical examination should be performed. Strengthening health knowledge, health education and publicity of hypertension are also critical for children hypertension.

\section{Acknowledgments}

Funding: None.

\section{Footnote}

Reporting Checklist: The authors have completed the STROBE reporting checklist. Available at http://dx.doi. org/10.21037/tp-20-446

Data Sharing Statement: Available at http://dx.doi. org/10.21037/tp-20-446

Conflicts of Interest: All authors have completed the ICMJE uniform disclosure form (available at http://dx.doi. org/10.21037/tp-20-446). The authors have no conflicts of interest to declare.

Ethical Statement: The authors are accountable for all aspects of the work in ensuring that questions related to the accuracy or integrity of any part of the work are appropriately investigated and resolved. All procedures performed in this study involving human participants were in accordance with the Declaration of Helsinki (as revised in 2013). The study was approved by the ethics committee of The Second Affiliated Hospital and Yuying Children's Hospital (LCKY2018-79). Individual consent for this retrospective analysis was waived.

Open Access Statement: This is an Open Access article distributed in accordance with the Creative Commons Attribution-NonCommercial-NoDerivs 4.0 International License (CC BY-NC-ND 4.0), which permits the noncommercial replication and distribution of the article with the strict proviso that no changes or edits are made and the original work is properly cited (including links to both the formal publication through the relevant DOI and the license). See: https://creativecommons.org/licenses/by-nc-nd/4.0/.

\section{References}

1. Flynn JT, Kaelber DC, Baker-Smith CM, et al. Clinical Practice Guideline for Screening and Management of High Blood Pressure in Children and Adolescents. Pediatrics 2017;140:e20171904.

2. Riley M, Hernandez AK, Kuznia AL. High Blood Pressure in Children and Adolescents. Am Fam Physician 2018;98:486-494.

3. Urbina EM, Khoury PR, McCoy C, Daniels SR, Kimball TR, Dolan LM. Cardiac and vascular consequences of pre-hypertension in youth. J Clin Hypertens (Greenwich) 2011;13:332-42.

4. Ukoh UC, Ujunwa FA, Muoneke UV, et al. Oscillometric blood pressure profile of adolescent secondary school students in Abakaliki metropolis. Ann Afr Med 2020;19:31-39.

5. Revision Committee of Chinese Guidelines for the Management of Hypertension. Chinese guidelines for the management of hypertension (2018 revised edition). Cardio-cerebrovascular Disease Prevention and Treatment 2019;19:1-44.

6. Hanevold CD. White Coat Hypertension in Children and Adolescents. Hypertension 2019;73:24-30.

7. Weaver DJ Jr. Hypertension in Children and Adolescents. Pediatr Rev 2017;38:369-382.

8. Litwin M, Michałkiewicz J, Gackowska L. Primary hypertension in children and adolescents is an immunometabolic disease with hemodynamic consequences. Curr Hypertens Rep 2013;15:331-9.

9. Oh JH, Hong YM. Blood Pressure Trajectories from Childhood to Adolescence in Pediatric Hypertension. Korean Circ J 2019;49:223-37.

10. Nardin C, Maki-Petaja KM, Miles KL, et al. Cardiovascular Phenotype of Elevated Blood Pressure Differs Markedly Between Young Males and Females: The Enigma Study. Hypertension 2018;72:1277-84.

11. Ezeudu CE, Chukwuka JO, Ebenebe JC, et al. Hypertension and prehypertension among adolescents attending secondary schools in urban area of South-East, Nigeria. Pan Afr Med J 2018;31:145.

12. Staessen JA, Wang J, Bianchi G, et al. Essential 
hypertension. Lancet 2003;361:1629-41.

13. Shankar RR, Eckert GJ, Saha C, et al. The change in blood pressure during pubertal growth. J Clin Endocrinol Metab 2005;90:163-7.

14. Abdul-Razak S, Daher AM, Ramli AS, et al. Prevalence, awareness, treatment, control and socio demographic determinants of hypertension in Malaysian adults. BMC Public Health 2016;16:351.

15. Daştan İ, Erem A, Çetinkaya V. Urban and rural differences in hypertension risk factors in Turkey. Anatol J Cardiol 2017;18:39-47.

16. Wang J, Sun W, Wells GA, et al. Differences in prevalence of hypertension and associated risk factors in urban and rural residents of the northeastern region of the People's Republic of China: A cross-sectional study. PLoS One 2018; 13:e0195340.

17. Bucher BS, Ferrarini A, Weber N, et al. Primary hypertension in childhood. Curr Hypertens Rep 2013;15:444-52.

18. Qiao S, Ye Q, Dou Y, et al. Analysis for hypertension and

Cite this article as: Wang Z, Shao Y, Jin J, Rong X, Qiu H, Wu R, Chu M. Clinical follow-up study of 166 cases of children with hypertension. Transl Pediatr 2021;10(7):1834-1842. doi: $10.21037 /$ tp-20-446 related risk factors of physical examination population. Int J Clin Exp Med 2013;6:785-93.

19. Sabri M, Gheissari A, Mansourian M, et al. Essential hypertension in children, a growing worldwide problem. J Res Med Sci 2019;24:109.

20. Pocobelli G, Dublin S, Enquobahrie DA, et al. Birth Weight and Birth Weight for Gestational Age in Relation to Risk of Hospitalization with Primary Hypertension in Children and Young Adults. Matern Child Health J 2016;20:1415-23.

21. Pluymen LP, Smit HA, Wijga AH,et al. Cesarean Delivery, Overweight throughout Childhood, and Blood Pressure in Adolescence. J Pediatr 2016;179:111-7.e3.

22. Charles L, Triscott J, Dobbs B. Secondary Hypertension: Discovering the Underlying Cause. Am Fam Physician 2017;96:453-61.

23. Bulpitt CJ. Blood urea changes in hypertensive patients according to therapy given, blood pressure control, and serum potassium levels. Br Heart J 1974;36:383-6. 